\title{
Modified Simplex Splitting Algorithm for Finding Feasible Solution of Systems of Linear Inequalities
}

\author{
E. O. Effanga \\ Department of Mathematics/Statistics and Comp. Science, University of Calabar \\ P. M. B. 1115, Calabar, Cross River State, Nigeria \\ E-mail: effanga2005@yahoo.com \\ Z. Lipcsey \\ Department of Mathsematics/Statistics and Comp. Science, University of Calabar \\ P. M. B. 1115, Calabar, Cross River State, Nigeria \\ E-mail: zlipcsey@yahoo.com \\ M. E. Nja (Corresponding author) \\ Department of Mathsematics/Statistics, Cross River State University of Technology \\ Calabar, Cross River State, Nigeria \\ E-mail: mbenja@yahoo.com
}

Received: August 22, 2011 Accepted: September 21, 2011 Published: February 1, 2012

doi:10.5539/jmr.v4n1p116 URL: http://dx.doi.org/10.5539/jmr.v4n1p116

\begin{abstract}
In this paper the existing simplex splitting algorithm for finding a feasible solution of systems of linear inequalities is modified by evolving a vertex-determination technique. The existing algorithm cannot determine when the system of linear inequalities is infeasible hence the need for a modification. The modified algorithm is able to determine the feasible solution whenever it exists and to detect infeasibility whenever it occurs. The modified algorithm is tested on a problem that has a feasible solution and also on a problem that has no feasible solution and is found to work perfectly well.
\end{abstract}

Keywords: Feasible solution, Infeasible solution, Polynomially bounded algorithm, Generalized relaxation method, Simplex splitting algorithm

\section{Introduction}

A number of methods exist for finding solutions to systems of linear inequalities. These include the relaxation or projection method developed by Agmon (1954), generalized relaxation method developed by Motzkin and Schonberg (1954), the ellipsoid method by Khachiyan (1979) and the simplex splitting algorithm developed by Levin and Yamnitsky (1982).

Among all these methods, the ellipsoid and the simplex splitting methods solve the problem in polynomial time. One major disadvantage of the ellipsoid method is that it is a nonlinear structure being used in solving a linear problem. Also it takes a very long time for the ellipsoid method to detect nonexistence of solution.

The simplex splitting method involves arbitrary selection of initial simplex with $(n+1)$ vertices for the problem involving $n$ variables, large enough to include at least one feasible solution (if any). If the centre of the simplex is a feasible solution of the system of linear inequalities, then the problem is solved, otherwise the procedure continues until a desired solution is obtained. Although the existing algorithm is polynomially bounded it has some pitfalls, namely, the arbitrary choice of the vertices of the initial simplex, and its inability to detect when the problem is infeasible. Effanga (2009), proffered a solution to this problem by modifying the existing simplex splitting algorithm.

\section{The Problem}

The problem to be solved is the following system of strict linear inequalities.

$$
\sum_{j=1}^{n} a_{i j} x_{j}<b_{i}, i=1,2, \cdots, m
$$




$$
x_{j}>0, j=1,2, \cdots, n \text {. }
$$

If the given system of linear inequalities is not strict, they can be made to be strict by adding a very small positive number $\varepsilon$ to $b_{i}$ for $i=1,2, \cdots, m$, see Eiselt, et al (1987).

\section{The Existing Simplex Splitting Algorithm}

The outline of steps is as follows:

Step 0 Select the initial simplex with vertices $v^{1}, v^{2}, \cdots, v^{n+1}$ such that it include at least one feasible point (if any), and compute the centre of the simplex as follows

$$
x^{0}=\frac{1}{n+1} \sum_{i=1}^{n+1} v^{\prime} .
$$

Set $k=0$

Step 1 Is inequality satisfied?

Yes: Stop, $x^{k}$ is a solution to the problem.

No: Select the $i^{\text {th }}$ constraint such that

$$
A_{i} x^{k} \geq b, i=1,2, \cdots, m \text {. }
$$

Step 2 Calculate the slack $S_{i}\left(v^{l}\right)$ of the $l^{\text {th }}$ vertex with respect to the $i^{\text {th }}$ constraint as

$$
S_{i}\left(v^{l}\right)=A_{i}\left(x^{k}-v^{l}\right), l=1,2, \cdots, n+1
$$

and determine $\mathrm{p}$ such that

$$
S_{i}\left(v^{p}\right)=\max _{l}\left\{S_{i}\left(v^{l}\right)\right\}
$$

Step 3 Calculate

$$
\lambda_{l}=1-\frac{S_{i}\left(v^{l}\right)}{n^{2} S_{i}\left(v^{l}\right)}, l=1,2, \cdots, n+1
$$

and determine the vertices of the new simplex as

$$
v^{l}=v^{p}+\frac{1}{\lambda_{l}}\left(v^{l}-v^{p}\right), l=1,2, \cdots, n+1
$$

Step 4 Compute the centre of the new simplex as

$$
x^{k+1}=\frac{1}{n+1} \sum_{l=1}^{n+1} v^{l}
$$

Set $k=k+1$. Return to Step 1 .

\section{Selection of Vertices for the Initial Simplex}

Let $v_{0}:=(0,0,0, \ldots, 0,0), v_{1}:=(1,0,0, \ldots, 0,0), v_{2}:=(0,1,0, \ldots, 0,0), \cdots, v_{n}:=(0,0,0, \ldots, 0,1)$ be $(n+1)$ vectors, with $v_{1}, v_{2}, \cdots, v_{n}$ independents.

Let

$$
\begin{gathered}
C=\left\{x: x=\sum_{j=1}^{n} \lambda_{j} v_{j}, 0 \leq x_{j}<\infty, j=1,2, \cdots, n\right\} \\
S_{0}:=\operatorname{Co}\left\{v_{j}\right\}_{j=0}^{n}=\left\{x: x=\sum_{j=0}^{n} \lambda_{j} v_{j}, \sum_{j=0}^{n} \lambda_{j}=1, \lambda_{j} \geq 0, j=0,1,2, \cdots, n\right\} \\
S_{00}:=\operatorname{Co}\left\{v_{j}\right\}_{j=1}^{n}=\left\{x: x=\sum_{j=1}^{n} \lambda_{j} v_{j}, \sum_{j=1}^{n} \lambda_{j}=1, \lambda_{j} \geq 0, j=1,2, \cdots, n\right\}
\end{gathered}
$$

Lemma 1

$$
S_{0}=\bigcup_{t=0}^{I} t S_{00}=\bigcup_{t=0}^{I} \bigcup_{x \in S_{00}} t x
$$


Proof: (i) Let $y \in S_{0}$. Then $y \neq 0$ and

$$
\begin{aligned}
& y=\sum_{j=0}^{n} \lambda_{j} v_{j}=\lambda_{0} v_{0}+\sum_{j=1}^{n} \lambda_{j} v_{j}=\sum_{j=1}^{n} \lambda_{j} v_{j}\left(\text { since } v_{0}=0\right) \\
& \sum_{j=1}^{n} \lambda_{J} \leq 1 .
\end{aligned}
$$

Let

$$
\sum_{j=1}^{n} \lambda_{j}=: t
$$

if $t>0$, then

$$
y=t \sum_{j=1}^{n} \frac{\lambda_{j} v_{j}}{t}=t x,
$$

where $x=\sum_{j=1}^{n} \frac{\lambda_{j} v_{j}}{t} \in S_{00}$ (since $\sum_{j=1}^{n} \frac{\lambda_{j}}{t}=1$ and $0<t \leq 1$ ).

(ii) let $y=t x$, where $x \in S_{00}$ and $0 \leq t \leq 1$

if $t>0, x=\sum_{j=1}^{n} \lambda_{j} v_{j}$ with $\sum_{j=1}^{n} \lambda_{j}=1$ and $\lambda_{j} \geq 0$, then $y=\sum_{j=1}^{n} t \lambda_{j} v_{j}+(1-t) v_{0}=\sum_{j=1}^{n} \mu_{j} v_{j}+\mu_{0} v_{0}=\sum_{j=0}^{n} \mu_{j} v_{j}$, where $\mu_{0}=1-t$ and $\mu_{j}=t \lambda_{j}, j=1,2, \cdots, n$.

If $t=0, y=0 \cdot x, \forall x \in S_{00}, \mu_{j}=0, j=1,2, \cdots, n, \mu_{0}=1$.

$$
\sum_{j=0}^{n} \mu_{j}=\mu_{0}+\sum_{j=1}^{n} \mu_{j}=1-t+t=1, \mu_{j} \geq 0,0 \leq j \leq n .
$$

Hence, $y \in S_{0}$.

\section{Lemma 2}

$$
M S_{0}=\bigcup_{0 \leq t \leq M} t S_{00}=\bigcup_{0 \leq t \leq M} \bigcup_{x \in S_{00}} t x
$$

Proof: Multiplying equation 12 through by M gives

$$
M S_{0}=M \bigcup_{t=0}^{I} t S_{00}=\bigcup_{t=0}^{I} M S_{00}=\bigcup_{0 \leq t \leq M} r S_{00}
$$

with $r:=M t$.

Lemma 3 If $x \in C \backslash\{0\}$, then $x=t x^{\prime}$, where $x^{\prime} \in S_{00}$, and $t>0$. For an $x$, $t$ and $x^{\prime}$ are unique.

Proof: (i) Let $x=\sum_{j=0}^{n} \lambda_{j} v_{j}=\sum_{j=1}^{n} \lambda_{j} v_{j}+\lambda_{0} v_{0}=\sum_{j=1}^{n} \lambda_{j} v_{j}, \lambda_{j} \geq 0,1 \leq j \leq n$.

Let $t=\sum_{j=1}^{n} \lambda_{j}>0$, then $x=t \frac{\sum_{j=1}^{n} \lambda_{j} v_{j}}{t}=t \sum \lambda_{j}^{\prime} v_{j}=t x^{\prime}$. With $\sum_{j=1}^{n} \lambda_{j}^{\prime}=1$ and $x^{\prime}=\sum_{j=1}^{n} \lambda_{j}^{\prime} v_{j} \in S_{00}$.

(ii)Suppose $x=t_{1} x_{1}^{\prime}=t_{2} x_{2}^{\prime}$. Let $x_{1}^{\prime}=\sum_{j=1}^{n} \lambda_{j}^{\prime} v_{j} \in S_{00}$ and $x_{2}^{\prime}=\sum_{j=1}^{n} \mu_{j}^{\prime} v_{j} \in S_{00}$.

Then

$$
\begin{aligned}
t_{1} x_{1}^{\prime}=t_{2} x_{2}^{\prime} & \Rightarrow \sum_{j=1}^{n} t_{1} \lambda_{j}^{\prime} v_{j}=\sum_{j=1}^{n} t_{2} \mu_{j}^{\prime} v_{j} \\
& \Rightarrow t_{1} \lambda_{1}^{\prime}=t_{2} \mu_{2}^{\prime}, j=1,2, \cdots, n \\
& \Rightarrow t_{1} \sum_{j=1}^{n} \lambda_{j}^{\prime}=t_{2} \sum_{j=1}^{n} \mu_{j}^{\prime} \\
& \Rightarrow t_{1}=t_{2} .
\end{aligned}
$$

Hence $x_{1}^{\prime}=x_{2}^{\prime}$. 
Lemma 4 Let $f(x)=\sum_{j=1}^{n} x_{j}$, if $x=\sum_{j=1}^{n} x_{j} v_{j}$ and $x \neq 0$, then $\frac{x}{f(x)} \in S_{00}, \forall x \in C$.

Proof: If $x \neq 0$, then $f(x)=\sum_{j=1}^{n} x_{j}>0$. If $x=\sum_{j=1}^{n} x_{j} v_{j}$ then $\frac{x}{f(x)}=\frac{\sum_{j=1}^{n} x_{j} v_{j}}{f(x)} \in S_{00}\left(\right.$ since $\left.\sum_{j=1}^{n} \frac{x_{j}}{f(x)}=1\right)$.

Theorem 1 If a feasible region $F_{r}$ is compact, then $F_{r} \subseteq R S_{0}$, with $R=\max _{x \in F_{r}} f(x)$.

Proof: If $x \in F_{r}$ and $x=0$, then $x \in R S_{0}$; If $x \neq 0$, then by lemma 2 and lemma 4 and $f(x) \leq R, x=f(x) \frac{x}{f(x)} \in R S_{0}$. Hence $F_{r} \subseteq R S_{0}$.

Theorem 2 If a feasible region $F_{r}$ is bounded and if the constraints matrix contains a row $i *$ with all entries positive, then with,

$$
\begin{gathered}
R=\frac{\max _{1 \leq i \leq m}\left\{\left|b_{j}\right|\right\}}{M} \\
M=\min \left\{\min _{1 \leq j \leq n}\left\{a_{i \cdot j}: a_{i \cdot j}>0\right\}, \frac{1}{2}\right\} \\
F_{r} \subseteq R S_{0}
\end{gathered}
$$

The proof of Theorem 4.2 is provided in Effanga (2009).

Corollary The simplex with vertices at $V^{0}(0,0, \cdots, 0), V^{1}(R, 0, \cdots, 0), \cdots, V^{n}(0,0, \cdots, R)$ enclosed the feasible region if it is compact.

\section{Detection of infeasibility}

Theorem 3 The modified simples splitting algorithm will terminate after at most $6 n(n+1) \log _{2} R$ number of iterations with either a feasible solution to the linear programming problem or with an indication that no feasible solution exists.

Proof: In the ellipsoid method developed by Khachiyan(1979), the algorithm terminates after at most $6 n(n+1) L$ iterations, where $2^{L}$ is the radius of the initial ellipsoid with centre at the origin. But the vertices of the initial simplex are points on the ellipsoid of radius $R$. Thus by letting $R=2^{L}$, we obtain $L=\log _{2} R$.

Hence the modified simplex splitting algorithm will terminate after at most $6 n(n+1) \log _{2} R$ number of iterations.

\section{The Outline of the Modified Simplex Splitting Algorithm}

The outline of steps is as follows:

Step 0 Select the initial simplex with vertices $V^{0}(0,0, \cdots, 0), V^{1}(R, 0, \cdots, 0), \cdots, V^{n}(0,0, \cdots, R)$ such that it include the feasible region (if any), and compute the centre of the simplex as follows

$$
x^{0}=\frac{1}{n+1} \sum_{l=0}^{n} v^{l}
$$

Set $k=0$.

Step 1 Is $A x^{k}<b$ ?

Yes: Stop, $x^{k}$ is a solution to the problem.

No: Go to step 2.

Step 2 Is $k>6 n(n+1) \log _{2} R$ ?

Yes: Stop, no feasible solution exists.

No: Go to step 3.

Step 3 Select the $i^{\text {th }}$ constraint such that $A_{i} x^{k} \geq b_{i}, i=1,2, \cdots, m$

Go to step 4.

Step 4 Calculate the slack $S_{i}\left(v^{l}\right)$ of the $l^{\text {th }}$ vertex with respect to the $i^{\text {th }}$ constraint as $S_{i}\left(v^{l}\right)=A_{i}\left(x^{k}-v^{l}\right), l=1,2, \cdots, n$ and determine $\mathrm{p}$ such that $S_{i}\left(v^{p}\right)=\max _{l}\left\{S_{i}\left(v^{l}\right)\right\}$.

Step 5 Calculate $\lambda_{l}=1-\frac{S_{i}\left(v^{l}\right)}{n^{2} S_{i}\left(v^{l}\right)}, l=1,2, \cdots, n$ and determine the vertices of the new simplex as $v^{l}=v^{p}+\frac{1}{\lambda_{l}}\left(v^{l}-v^{p}\right)$, $l=1,2, \cdots, n$.

Step 6 Compute the centre of the new simplex as $x^{k+l}=\frac{1}{n+1} \sum_{l=0}^{n} v^{l}$.

Set $k=k+1$. Return to Step 1 . 


\section{Illustration}

Example 1 Consider the following system of linear inequalities,

$$
\begin{gathered}
5 x_{1}-4 x_{2} \leq 14 \\
-x_{1}+4 x_{2} \geq 2 \\
2 x_{1}+x_{2} \geq 5 \\
6 x_{1}-x_{2} \geq 3 \\
4 x_{1}+9 x_{2} \leq 60 \\
x_{1} \geq 0, x_{2} \geq 0 \\
R=\frac{\max \left\{b_{i}\right\}}{M}=\frac{60}{\frac{1}{2}}=120
\end{gathered}
$$

If the problem has a feasible solution, then it is sure that the vertices of the simplex that enclosed the feasible region are $A(0,0), B(120,0)$ and $C(0,120)$. See figure 1 . The modified simplex splitting algorithm gives the feasible solution as $x_{1}=4.4265, x_{2}=3.7511$.

$<$ Figure 1>

Example 2 Consider the following system of linear inequalities,

$$
\begin{gathered}
2 x_{1}+x_{2} \leq 2 \\
3 x_{1}+4 x_{2} \geq 12 \\
x_{1} \geq 0, x_{2} \geq 0 \\
R=\frac{\max \left\{b_{i}\right\}}{M}=\frac{24}{\frac{1}{2}}=48 .
\end{gathered}
$$

$<$ Figure 2>

Clearly the simplex with vertices $A(0,0), B(24,0)$ and $C(0,24)$ does not enclosed the feasible region as it does not exists. See figure 2. The modified simplex splitting algorithm confirms that this problem does not have a feasible solution.

\section{Concluding Remarks}

The modified simplex splitting algorithm presented in this paper is designed for the system of strict linear inequalities of less than type. Hence any inequality not in this form must first undergo suitable transformations before applying the algorithm. A very large simplex enclosing the feasible region may slow down the rate of convergence of the algorithm. The simplex selected to enclose the feasible region discussed in section 4 may not, in all cases, be the convex hull of the feasible region and thus required further investigation.

\section{References}

E. O. Effanga. (2009). Alternative Technique for solving linear programming problems, Ph. D Thesis. Department of Mathematics/Statistics and Computer Science, University of Calabar - Nigeria (unpublished).

G. Eiselt, C. Pederzolli, \& L. Sandblom. (1987). Operations Research. W de G. New York.

L. A. Levin, \& B. Yammitsky. (1982). An old linear programming algorithm runs in polynomial time. Proceeding $23^{\text {rd }}$ Annual Symposium of foundations of Computer Science. IEEE Computer Society, Long Beach. California, 327 - 328.

L. G. Khachiyan. (1979). Polynomial algorithm for linear programming. Doklady Academic Nauk SSSR 255/5, 1093 1096.

S. Agmon. (1954). The relaxation method for linear complementarity problems. Mathematical Programming 28, 349 362.

T. S. Motzkin, \& I. J. Schonberg. (1954). The relaxation method for linear inequalities. Canadian Journal of Mathematics 2, $293-404$. 